\title{
Pressions Anthropiques Et Dynamique D’occupation Des Terres Dans Le Terroir De Ziguéna, Zone Cotonnière Du Mali
}

\begin{abstract}
Abdou Ballo
Département de Géographie, Faculté d’Histoire et de Géographie, Université des Sciences Sociales et de Gestion de Bamako, Mali

\section{Souleymane Sidi Traoré}

Unité Système d’Information Géographique et Télédétection, Labosep, CRRA de Sotuba, IER, Bamako, Mali
\end{abstract}

\section{Baba Coulibaly}

Département de Géographie, Faculté d’Histoire et de Géographie, Université des Sciences Sociales et de Gestion de Bamako, Mali

\section{Cheick Hamalla Diakité}

Moriké Diawara

Alou Traoré

Unité Système d’Information Géographique et Télédétection, Labosep, CRRA de Sotuba, IER, Bamako, Mali.

\section{Sidi Dembélé}

Département de Géographie, Faculté d'Histoire et de Géographie, Université des Sciences Sociales et de Gestion de Bamako, Mali

\section{Abstract}

In Ziguéna terroir, the combined effects of drought and anthropogenic actions led to the widespread degradation of vegetation cover and of land. This work aimed at characterizing the dynamics of land use and land cover in relation to anthropogenic pressures in Ziguéna terroir. The methodology consisted in identifying and characterizing land use and land cover classes. Landsat images for the years 1986 and 2013 and population data for the years 1987, 1998 and 2009 were used. Visual interpretation of the images and post-classification comparison of the results were used to generate land use and land cover classes and calculate their rate of change. The results reveal that the natural vegetation has lost $55 \%$ of its original coverage (1514.3 ha) between 1987 and 2013. During the same period, the agricultural area increased by $47 \%$ (1608 ha). The projection of land use and land cover classes predicted an increase of agricultural land of about $34.60 \%$ by year 
2030 compared to its coverage of year $2013(+1191.03 \mathrm{ha})$ at the expense of natural vegetation which will lose about $40.63 \%$ of its coverage $(-1121.70$ ha). The dynamics of agricultural land is strongly linked to population growth rates with a correlation coefficient $r$ equal to 0.99 . This confirms a strong anthropogenic influence on land use and land cover dynamics. The results show the usefulness of remote sensing for mapping land use and land cover. Nevertheless it would be interesting to take into account the socioeconomic aspects for proper understanding of the dynamics.

Keywords: Dynamics, Anthropogenic pressures, Land use and land cover

\section{Résumé}

Dans le terroir de Ziguéna, les effets conjugués de la sécheresse et des actions anthropiques ont entraîné la réduction généralisée du couvert végétal et la dégradation des terres. Ce travail avait pour objectif de caractériser la dynamique de l'occupation des terres en relation avec les pressions anthropiques dans le terroir de Ziguéna. La méthodologie adoptée a consisté à l'identification et à la caractérisation des unités d'occupation du sol. Les images Landsat de 1986 et de 2013 ainsi que des données de population de 1987; 1998 et 2009 ont été utilisées. La méthode d'interprétation visuelle des images et la comparaison des résultats ont été utilisées pour dégager les unités d'occupation et calculer leur taux de changement. L'analyse des résultats révèle que la végétation naturelle a perdu 55\% de sa superficie initiale (1514,3 ha) entre 1987 et 2013. Durant la même période, la zone agricole a augmenté de 47\% (1608 ha). La projection des unités d'occupation du sol prévoit une augmentation des superficies agricoles de 34,60\% par rapport à sa superficie de 2013 (+1191,03 ha) au dépend de la végétation naturelle qui perdra environ $40,63 \%$ de sa superficie (-1121,70 ha) à l'horizon 2030. La dynamique des terres agricoles est fortement liée au taux de croissance démographique avec un coefficient de corrélation r:0,99. Ceci confirme une forte influence anthropique sur l'évolution de l'occupation des terres. Les résultats montrent l'utilité de la télédétection pour la cartographie de l'occupation des terres, néanmoins il serait intéressant de prendre en compte les aspects socio-économiques pour une bonne appréhension de la dynamique.

Mots-clés : Dynamique, Pressions anthropiques, Occupation du sol

\section{Introduction}

$\mathrm{Au}$ cours de ces dernières décennies, les activités humaines en particulier l'agriculture ont conduit à la transformation progressive d'une très grande partie de la surface terrestre. Dans la zone cotonnière du Mali cette 
pression anthropique est relativement importante à cause d'un essor de la culture cotonnière et de l'élevage depuis trois décennies (Cuny et Sorg, 2003). La zone occupe l'angle Sud-Est à Sud-Ouest du pays et est frontalière du Burkina Faso, de la Cote d'Ivoire et de la Guinée (Benjaminsen et Sjaastad, 2002).

La production du coton a gagné une grande importance dans la zone au cours des dernières années causant une forte densification de la population surtout dans sa partie sahélienne. La population s’accroit rapidement depuis plusieurs décennies accentuant ainsi la pression humaine sur le milieu naturel. Ce processus rapide a eu des effets importants sur l'occupation des terres. La superficie des champs cultivés a augmenté continuellement au cours des cinquante dernières années (Cuny et Sorg, 2003).

L’expansion territoriale agricole a été importante de même que la croissance démographique. Cette dernière s'est intensifiée à partir des années 1980 (avec un taux de près de 4\%), témoignant d'un fort dynamisme économique induit par la production cotonnière (CMDT, 1998). Le nombre d'exploitations agricoles et leur taille ont sensiblement augmenté et, en 1998, l'espace agricole est quasi saturé dans la plupart des terroirs (Cuny et Sorg, 2003). Pendant la période 1952-1990, le rythme annuel de défrichement agricole a été de $6 \%$, provoquant une régression considérable de l'espace forestier dont les jachères (Cuny et Sorg, 2003).

La mise en relation de l'évolution démographique et des besoins en terres cultivables permet donc de poser le problème de la disponibilité en terre pour les générations à venir (Adam et Pofadji, 2008). Des études corrélant la disponibilité et l'usage des ressources naturelles avec l'évolution démographique sont rares. Aussi, l'appréciation des changements d'états des unités d’occupation du sol et sa relation avec l'évolution démographique s'avèrent être nécessaires. Ce travail se propose de combler ce déficit d'information en caractérisant la dynamique de l'occupation des terres en relation avec les pressions anthropiques dans le terroir de Ziguéna.

\section{Matériels et Méthodes}

\section{Présentation du site d'étude}

L'étude a été conduite dans le terroir de Ziguéna ( $5^{\circ} 50^{\prime \prime}$ Ouest à $5^{\circ} 55^{\prime \prime}$ Ouest et $11^{\circ} 35^{\prime \prime}$ Nord à $11^{\circ} 41^{\prime \prime}$ Nord) dans la zone cotonnière du Mali (Figure 1). Le terroir couvre une superficie d'environ $69 \mathrm{~km}^{2}$ et est caractérisé par un climat de type Soudanien avec des moyennes annuelles de pluie oscillant autour de 922 mm. L’année est divisée en deux saisons. La première est la saison des pluies de mai à octobre. Le mois le plus pluvieux est août avec des pluviométries moyennes de $200 \mathrm{~mm}$. La seconde est la saison sèche de novembre à avril. Elle commence avec le début de 
l'harmattan (vent sec frais et poussiéreux). Le relief est relativement accidenté avec des plateaux cuirassés principalement dans la partie SudOuest du terroir. La végétation naturelle est dominée par les savanes et est riche en essences floristiques. Le terroir est drainé par deux rivières qui alimentent un important bas-fond d'inondation dans la partie nord du terroir. Selon les résultats du recensement général de la population et de l'habitat de 2009, le village de Ziguéna était peuplé de 1221 habitants (RGPH, 2009). La population est composée majoritairement de Senoufo, Minianka, Bobo (agriculteurs), et Peulh (éleveurs). L'agriculture qui porte en grande partie sur le coton, le mil, le maïs, le riz et la pomme de terre constitue l'activité dominante de la population.

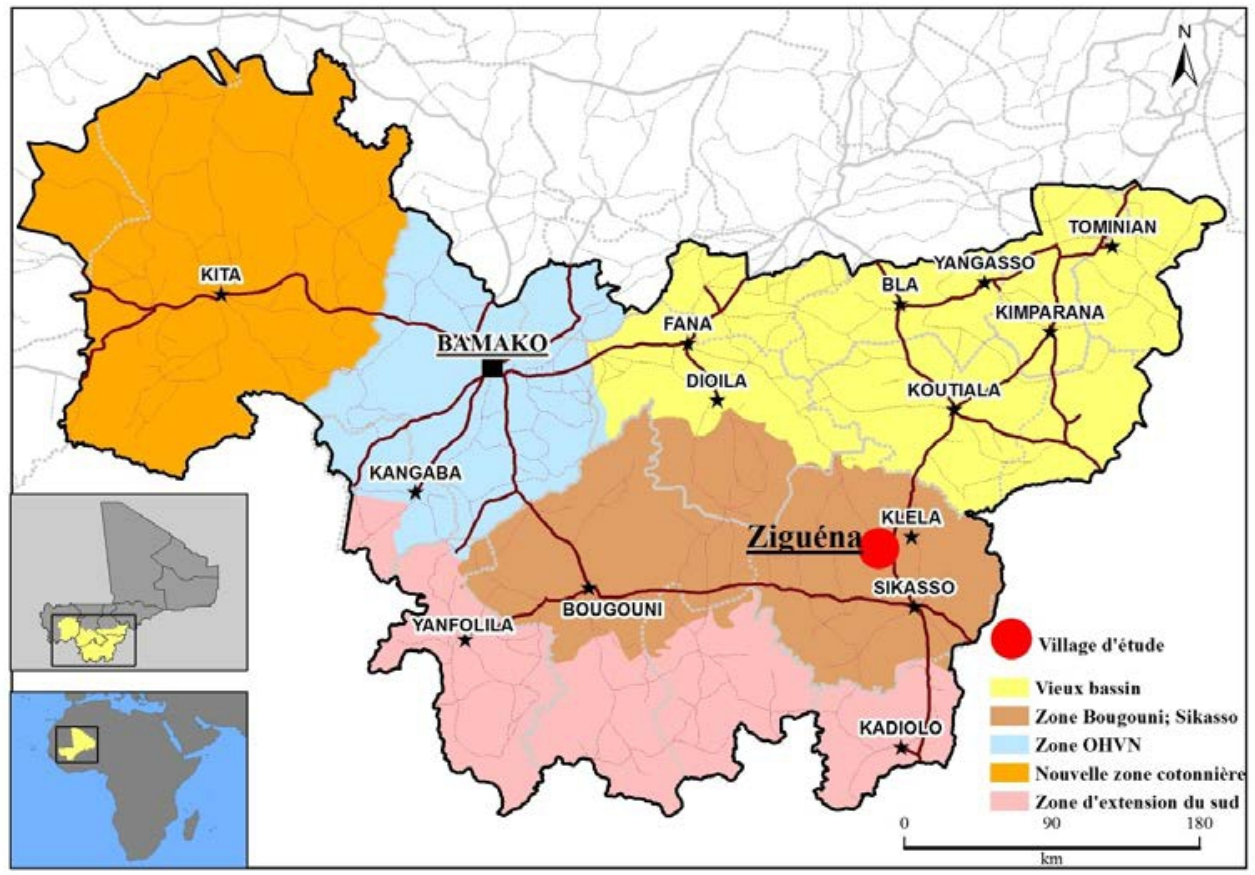

Figure 1: Localisation du terroir de Ziguéna dans la zone cotonnière du Mali

\section{Détection du changement d'occupation du sol}

Deux scènes d'image Landsat, d'une résolution de 30-m pixel, ont été utilisées dans le cadre de ce travail pour déterminer la dynamique de l'occupation du sol. Il s'agit d'une image Landsat TM (Thematic Mapper) acquise le 16 octobre 1986 et une image Landsat 8 OLI (Opérationnel Land Imager) du 30 septembre 2013. Ces images ont été géo-référencées en utilisant un polynôme de $1^{\mathrm{er}}$ degré du système UTM (Universal Transverse Mercator) zone 30 Nord. Des données de références sur l'occupation du sol ont été collectées lors de la visite de terrain effectuée dans la zone en octobre 2014. La méthode d'interprétation visuelle des images, qui permet de 
caractériser les principaux objets présents : sols, végétations, eaux, etc., d'une manière fidèle, systématique et exhaustive par leur comportement spectral, a été adoptée pour la cartographie de l’occupation des sols (Girard, 1995).

\section{Dynamique de la population}

$\mathrm{Au}$ Mali, le premier recensement général de la population et de l'habitat a été effectué en 1976, et depuis les recensements sont effectués approximativement tous les 10 ans (1976, 1987, 1998 et 2009). Ce travail a utilisé les données de ces recensements pour les années 1986, 1998 et 2009. La population des périodes intermédiaires (1992, 2003 et 2014), a été estimée en utilisant la formule suivante:

$$
P_{o+t}=P_{o}(1+\tau)^{n}
$$

Où $\boldsymbol{P}_{\boldsymbol{o}+\boldsymbol{t}}$ est la population estimée à partir du recensement précédent, $\boldsymbol{P}_{\boldsymbol{o}}$ est la population totale à l'année du recensement, $\boldsymbol{\tau}$ est le taux d'accroissement naturel, 1 est la constante et $\boldsymbol{n}$ : nombre d'année d'intervalle à partir du dernier recensement.

\section{Analyses des données}

La cartographie de l’occupation du sol a été réalisée sur deux dates: 1986 et 2013 pour le terroir d'étude. La méthode de comparaison des résultats post traitement (Hakan et al. 2009) a été utilisée pour déterminer la dynamique et le taux de changement par classe d'occupation durant la période d'étude. La projection linéaire des superficies en se basant sur le taux de changement annuel a été utilisée pour projeter dans le temps la dynamique future de l'occupation des sols dans la zone d'étude. La relation entre la superficie des terres cultivées-population d'une part et la relation végétation-population d'autre part ont été établies en utilisant la corrélation de Pearson (Traore et al. 2015). Enfin la pression foncière a été évaluée en utilisant l'indice agro-démographique qui est le ratio entre les terres cultivables disponibles et la population totale agricole (Djaouga et al. 2008).

\section{Résultats et Discussion \\ Dynamique de l'occupation du sol}

Les classes ou catégories d'occupation des sols se réfèrent à la classe dominante dans un polygone délimité. Les quatre (4) classes présentées dans le tableau (1) ont été utilisés comme nomenclature de référence pour ce travail. Il s’agit de la zone agricole, de la végétation, de la zone d’habitation et de l'eau. 
Tableau 1: Description des classes d'occupation des sols utilisées dans ce travail

\begin{tabular}{|c|c|}
\hline Classes & Définition \\
\hline Agricole & $\begin{array}{l}\text { Espaces cultivés avec ou sans arbres éparses (couverture de la canopée de } \\
20 \% \text { environ). Ces espaces sont occupés par des cultures annuelles } \\
\text { (principalement coton, mil, sorgho et maïs), récoltées en octobre-novembre, } \\
\text { puis les sols sont nus avec des résidus de cultures à partir de décembre. }\end{array}$ \\
\hline Végétation & $\begin{array}{l}\text { Classe mixte de terrain avec une végétation herbacée et une couverture } \\
\text { ligneuse. }\end{array}$ \\
\hline Habitat & $\begin{array}{l}\text { Zone principalement couverte d'établissement humain ou zone d’habitation } \\
\text { qui comporte souvent des portions de sol nu ou de végétation entre les bâtis. }\end{array}$ \\
\hline Eau & $\begin{array}{c}\text { Cours d'eau temporaire ou permanent tel que les rivières, les bas-fonds } \\
\text { inondés ou les ravins. }\end{array}$ \\
\hline
\end{tabular}

Les résultats du traitement des images (Tableau 2) montrent un changement significatif de l'occupation des sols durant la période d'étude. En 1986 la classe d'occupation dominante était la végétation qui occupait environ $68 \%$ du terroir soit 4275,00 ha. Elle était suivie de la zone agricole avec 29\% (1834,50 ha), la superficie en eau avec 3\% (184,00 ha) et la zone d'habitation qui couvrait à peine $0,10 \%$ du terroir avec 6,30 ha. Par contre en 2013, la classe d'occupation dominante était la zone agricole qui couvrait environ $55 \%$ de la superficie du terroir soit 3442,20 ha. La végétation constituait la seconde classe dominante et occupait $44 \%$ du terroir soit 2760,70 ha; la surface en eau et la zone d'habitation couvraient respectivement environ $1 \%$ du terroir soit 62,40 ha et 34,30 ha.

Tableau 2: Changement de l'occupation du sol entre 1986 et 2013 dans le terroir de Ziguéna

\begin{tabular}{ccccc}
\hline Classes d'occupation & $\mathbf{1 9 8 7}$ & $\mathbf{2 0 1 3}$ & Différence & *Tca \\
\hline Agricole & 1834,50 & 3442,20 & 1607,93 & 0,93 \\
Végétation & 4275,01 & 2760,73 & $-1514,28$ & 0,97 \\
Eau & 184,04 & 62,36 & $-121,68$ & $-0,99$ \\
Bâtis & 6,31 & 34,33 & 28,01 & 0,80 \\
\hline
\end{tabular}

*Tca $=$ Taux de changement annuel

En général les résultats (Figure 2) montrent une augmentation des superficies agricoles et de la zone d'habitation au dépend de la végétation. Durant la période d'étude, la zone agricole a augmenté de 47\% par rapport à sa superficie initiale de 1986 soit un taux d'accroissement annuel de 0,93 (59,55 ha/an). La zone d'habitation quant à elle a augmenté de 81,60\% par rapport à sa superficie initiale de 1986 avec un taux annuel d'accroissement de 0,80 (1,03 ha/an). Par contre la végétation a perdu $54,90 \%$ de sa superficie initiale de 1986 avec un taux annuel de réduction de 0,98 (-56,09 ha/an). Cette tendance confirme le résultat de Ruelland et al. 2010 qui a observé une augmentation de la zone agricole dans la même zone agro écologique (soudano-sahélienne). Cette dynamique est la conséquence d'une forte conversion des superficies de végétation naturelle en zone de culture 
surtout de coton.

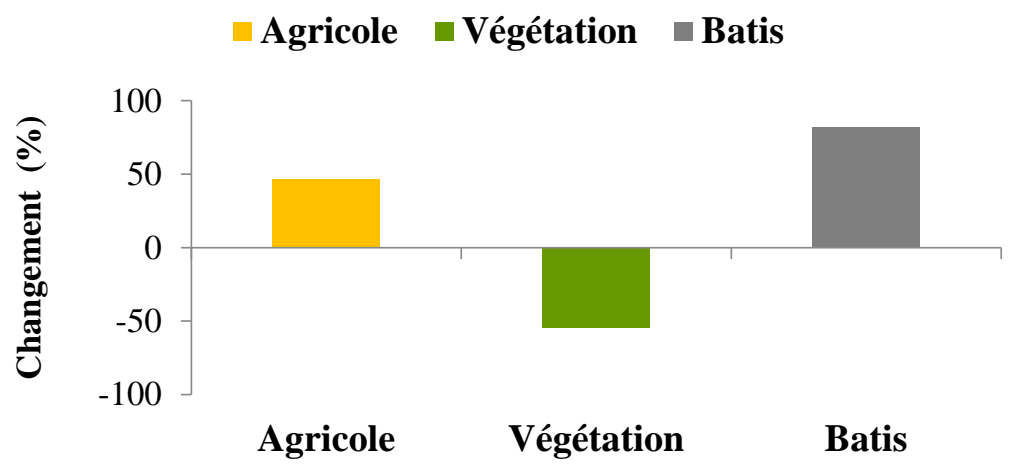

Figure 2: Dynamique de l’occupation du sol entre 1986 et 2013

\section{Relation entre la dynamique de la population et l'occupation du sol}

La corrélation de Pearson (Figure 3) révèle un lien entre la croissance de la population et la dynamique de l'occupation du sol. En général il existe une forte corrélation positive entre l'augmentation de la population et l'augmentation des superficies agricoles $\left(r^{2}: 0,99\right.$, p: 0,01) et la diminution du couvert végétal $\left(\mathrm{r}^{2}: 0,99 \mathrm{p}: 0,01\right)$. Cela signifierait que la croissance démographique a un impact négatif sur la dynamique de la végétation. L'expansion des superficies cultivées a certainement conduit à la diminution du couvert végétal. La forte corrélation négative entre la population et la végétation et la forte corrélation positive entre la population et la végétation sont imputables à un progrès faible en matière de technologies agricoles (Ouedrago et al. 2010). Il en ressort un défrichement progressif des surfaces en végétation pour augmenter la superficie afin d'augmenter la production. Cette situation est très commune des régions tropicales (Reenberg and Lund, 1998 ; Lambin et al. 2003 ; Ningal et al. 2008) et singulière dans la zone sud du Mali.

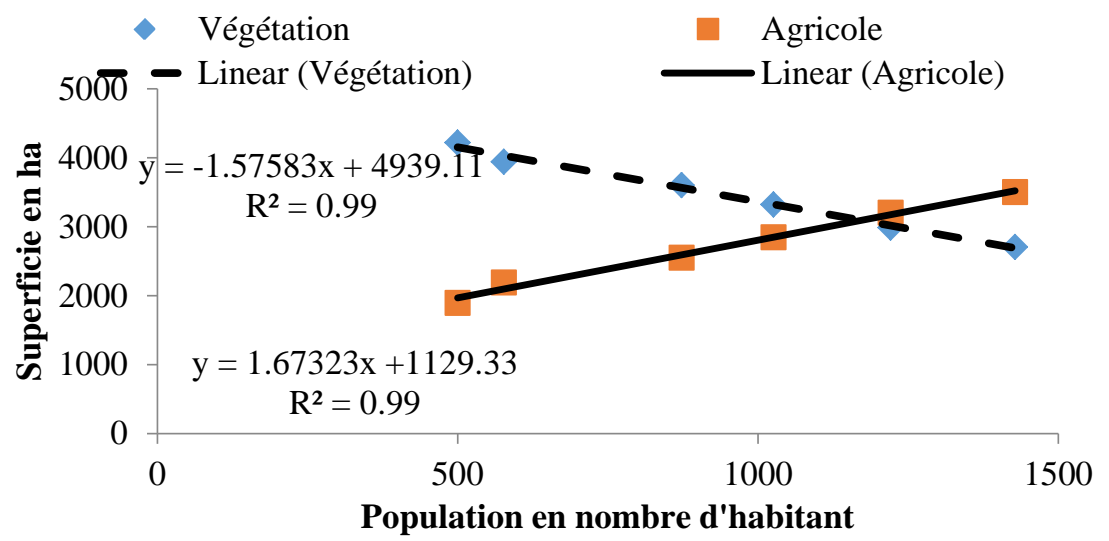

Figure 3 : Corrélations entre la population, la végétation et la superficie agricole 
Les résultats de ce travail ont aussi permis d'analyser et de dégager une tendance qui révèle l'augmentation des superficies agricoles $(47 \%)$ au détriment de la végétation naturelle qui a perdu $54,90 \%$ de sa superficie. Durant la période d'étude la population du terroir a triplé alors que la superficie agricole a doublée et le ratio superficie agricole par individu (indice agro-démographique, IAD) est passé de 3,8 ha par individu à 2,4 ha par individu (Figure 4). Ce qui dénote une forte pression de la population sur la végétation en termes de forte dynamique agricole. Le taux de conversion de la végétation en zone agricole, calculé à partir des résultats, a indiqué une augmentation annuelle de la zone agricole de 60 ha et une réduction annuelle de la végétation de 56 ha. Par ailleurs en 2007 la zone agricole a atteint la végétation en superficie, ce qui posera dans un futur proche un problème de disponibilité de terre agricole et diminuera fortement le ratio individusuperficie agricole. Avec ce taux d'évolution et en se basant sur l'hypothèse que cela continuera dans le temps et dans l'espace, les superficies agricoles augmenteront de 34,60\% par rapport à celle de 2013 soit 1191,03 ha au dépend de la végétation naturelle. Cette dernière perdra environ 40,63\% de sa superficie soit 1121,70 ha à l'horizon 2030.

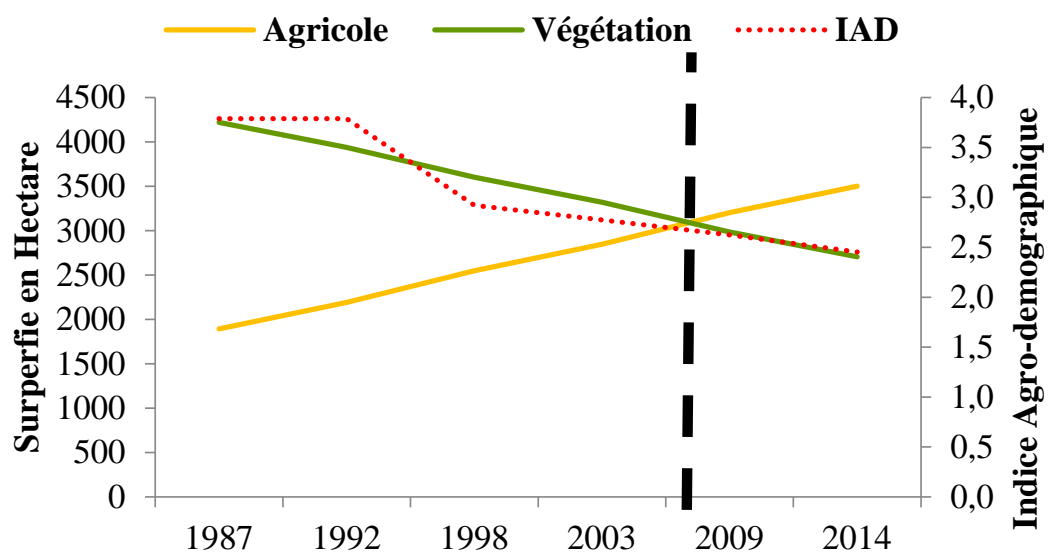

Figure 4: Evolution de la zone agricole et la végétation naturelle et de l'indice agrodémographique

\section{Conclusion}

La cartographie de la dynamique d'occupation du sol a été identifiée comme un des outils importants de la gestion environnementale. L'étude a permis d'analyser les différentes unités d'occupation du sol et leur état actuel et futur. L'analyse a révélé une tendance à la hausse des espaces cultivés au détriment de la végétation naturelle due à une forte pression anthropique. Ainsi la végétation a perdu -1514,28 ha entre 1986 et 2013 alors que la zone agricole a augmenté de son coté de 1607,93 ha. Ainsi le taux d'accroissement calculé sur la même période montre une augmentation annuelle de la zone 
agricole de 60 ha environ au dépend de la végétation qui perd environ 56 ha. Cette forte dynamique de l'augmentation de la superficie agricole et de diminution de la végétation est fortement liée à l'évolution démographique. En ouvre le ratio individu sur superficie agricole devient faible ce qui sous entend une tendance à la saturation de l'espace territorial. Une meilleure stratégie de gestion des ressources naturelles s’avère nécessaire pour que les générations actuelles et futures puissent jouir effectivement de ces ressources.

\section{Remerciements}

Les auteurs de cet article tiennent à remercier l'agence française de développement (AFD) qui a financé cette recherche dans le cadre du projet d'amélioration de la productivité dans la zone cotonnière du Mali (PASE II).

\section{References :}

Adam S., Pofadji M. (2008). Identification des espaces administratifs et de développement du Bénin : Approche géographique. Nouvelle Presse.

Benjaminsen, A., Sjaastad E., (2002). Géographie, transactions foncières et appropriation de la rente foncière dans la zone cotonnière du Mali, European Journal of Development Research, vol 14, num 2, pp 129-152.

CMDT, (1998). Rapport de Campagne Agricole 1997-1998. Bamako, Mali. Cuny P., Sorg J.P., (2003). Forêt et coton au sud du Mali: cas de la commune rurale de Sorobasso. Bois et Forêts des Tropiques, $\mathrm{N}^{\circ} 276$ (2) 17-30.

Djaouga, M., Houndagba, C., Chabi, B., Sinsin, B., (2008). Activités agropastorales, gestion des ressources naturelles et lutte contre la désertification dans la commune de Kalalé au Benin (Afrique), Acte de Communication au séminaire CTA Implications du changement climatique sur les systèmes de production agricole durable dans les ppays ACP. Quelles stratégies d'infiormation et de communication ? 26-31 octobre 2008, Ouagadougou, Burkina

Faso,

\section{ctaseminar2008.cta.int/pdf/Friday/Agropastoral.pdf.}

Girard, M., 1995. Apport de l'interprétation visuelle des images satellitaires pour l'analyse spatiale des sols, un exemple de la région de Lodève, Etude et Gestion des Sols, 2, 1, 7-24.

Hakan, A., Hakan, D., and Yüksel, I.U., (2009). Post-classification comparison of land covers using multi-temporal Landsat and ASTER imagery: the case of Kahramanmaraş, Turkey, Environmental Monitoring Assessment Journal, 151: 327-336.

Lambin, E.F., Geist, H.J., Lepers, E., (2003). Dynamics of land use and land cover change in tropical regions. Annual Review of Environment and resources 28: 205-241.

Ningal, T., Hartemink, A.E., Bregt, A.K., (2008). Land use change and 
population growth in the Morobe Province of Papua New Guinea between 1975 and 2000. Journal of Environmental Management 87: 117-124.

Ouedrago, I., Tigabu, M., Savadogo, P., Compaoré, H., Odén, P.C., and Ouadbra, J.M., (2010). Land cover change and its relation with population dynamics in Burkina Faso, West Arica. Land Degradation and Development, 20(2), 217-230, DOI: 10.1002/ldr.98.1.

Reenberg, A., Lund, C., (1998). Land use and land right dynamics determinants for resource management options in Eastern Burkina Faso. Human Ecology 26: 599-620.

RGPH, (2009). $4{ }^{\text {ème }}$ Recensement Général de la Population et de l'Habitat, INSAT-Mali, Résultats Définitifs Tome 0: Répertoire des villages, 318p.

Ruelland, D., Levavasseur, F., Tribotté, A., (2010). Patterns and dynamics of land-cover changes since the 1960s over three experimental areas in Mali, International Journal of Applied Earth Observation and Geoinformation,12, S11-S17.

Traore, S.S., Forkuo, E.K., Traore, P.C.S., Landmann, T., (2015). Assessing the inter-relationship between vegetation productivity, rainfall, population and land cover over the Bani River Basin in Mali (West Africa), IOSR Journal of Engineering, 05(06), 10-18. 